\title{
Making by grinding: Mechanochemistry boosts the development of halide perovskites and other multinary metal halides.
}

\author{
Francisco Palazon,* Yousra El Ajjouri, and Henk J. Bolink \\ Instituto de Ciencia Molecular, ICMol, Universidad de Valencia \\ C/ Catedrático J. Beltrán 2, 46980 Paterna, Spain \\ E-mail: Francisco.palazon@uv.es
}

Keywords: mechanochemistry, perovskites, solid-state, synthesis, ball-milling.

\begin{abstract}
Mechanochemical synthesis has recently emerged as a promising route for the synthesis of functional lead halide perovskites as well as other (lead-free) metal halides. Mechanochemical synthesis presents several advantages with regards to more commonly-used solution-based processes such as an inherent lower toxicity by avoiding organic solvents and a finer control over stoichiometry of the final products. The ease of implementation, either through the use of a simple mortar and pestle or with an electrically-powered ball-mill, and low amount of side products make mechanochemical synthesis appealing for upscaling the production of halide perovskites. Due to the defect tolerance of lead halide perovskites, they are ideally suited to be prepared by this solvent free method. However, the implementation of these semiconductors in in high-efficiency optoelectronic devices requires the transformation of synthesized powder into smooth thin films where still some hurdles remain to be solved.
\end{abstract}

\section{INTRODUCTION}

Mechanochemistry refers to the activation of chemical reactions triggered by mechanical energy. This energy might stem from several forces and operation modes such as impact, 
compression, or shear. In this review we will focus on mechanochemical synthesis ${ }^{1}$ (MCS) of compounds enabled by grinding or milling ${ }^{2}$ of their precursors, following the reaction:

$$
A(s)+B(s) \stackrel{M C S}{\longrightarrow} A B(s)
$$

MCS can be readily carried out by hand-grinding with mortar and pestle, by milling in shaking or planetary ball-mills or by other means such as mechanical grinding in twin-screw extruders. ${ }^{[1]}$ The exact operational mechanisms of MCS have not been completely elucidated. It is commonly accepted, though, that the reduction in particle size induced by grinding leads to the continuous exposure of "fresh" reactive surfaces that favors completion of the desired chemical reaction. $^{[1]}$ Despite its operational simplicity, mechanochemistry has long been overlooked in contrast to other synthetic methods such as, (green) solvent based synthesis, supercritical $\mathrm{CO}_{2}$ assisted chemistry or the use of ionic liquids. Nevertheless, in view of several recent reviews and perspectives it appears we are experiencing a renaissance of this underestimated field. One of the main benefits of MCS is that no solvents are needed. This is very relevant in the development of green chemistry as solvents typically represent important amounts of waste. Furthermore, many of them are hazardous for human health as well as for the environment. Therefore, MCS has recently been explored to synthesize a multitude of functional materials such as organic molecules, exfoliated graphene, metal-organic-frameworks, or metallic alloys. ${ }^{[1-9]}$

MCS has also been employed for the synthesis of different semiconductor materials such as transition metal chalcogenides. ${ }^{[10-12]}$ Nevertheless, as pointed out by Protesescu et al., ${ }^{[13]}$ the numerous structural defects that MCS causes in the rigid crystal lattices of traditional semiconductors such as CdSe or CdTe has been a major drawback until the advent of lead halide perovskites (LHPs). Crystal defects such as vacancies or interstitials frequently have energy

\footnotetext{
${ }^{1}$ Note that the term "mechanosynthesis", sometimes used as synonym of MCS refers to more specific reactions which happen upon recognition of macromolecules in certain geometric configurations, a process that mostly applies to biological processes.

2 Throughout this review, unless specified otherwise, "grinding” will be used as a generic term for both grinding (by hand) and milling.
} 
levels that are within the semiconductor bandgap. As such, they act as electron- or hole-traps, quenching the photoluminescence (increasing non-radiative recombination) and diminishing the overall semiconducting performances of the material (e.g., lower charge carrier diffusion lengths).

In the past decade, lead halide perovskites (LHPs) have re-emerged as a very promising class of semiconducting materials with excellent properties for photovoltaics and light-emission applications. ${ }^{[14,15]}$ One of the reasons for their success is the ease of forming high quality films from a wide range of processing methods such as single and dual step spin-coating, gas-assisted deposition, or thermal vacuum sublimation. ${ }^{[16-18]}$ The presence of grain boundaries, which inevitably are imperfect crystal domains, does not affect the semiconducting properties significantly, at least for the low-bandgap LHPs. This means that most structural defects are benign in terms of optoelectronics, as the energy levels introduced by them are either located outside of the bandgap (below the valence band or above the conduction band) or close to their edges (shallow traps). Hence, this peculiar property of LHPs makes them suitable, in principle, to be prepared by MCS. By grinding the perovskite precursor binary salts, the desired phase can be formed according to the following equation, where $\mathrm{A}$ is a monovalent cation (commonly methylammonium -MA; $\mathrm{CH}_{3} \mathrm{NH}_{3}{ }^{+}$-, formamidinium -FA; $\mathrm{CH}\left(\mathrm{NH}_{2}\right)_{2}{ }^{+}$, or cesium $-\mathrm{Cs}^{+}-$) and $\mathrm{X}$ a monovalent halide anion (commonly $\mathrm{I}^{-}, \mathrm{Br}^{-}$, or $\mathrm{Cl}^{-}$).

$$
A X(s)+P b X_{2}(s) \stackrel{M C S}{\longrightarrow} A P b X_{3}(s)
$$

Indeed, the MCS of LHPs and related compounds has been demonstrated by Stoumpos et al. as early as in 2013 by hand grinding with mortar and pestle. ${ }^{[19]}$ In this seminal report, the authors noted however that a subsequent thermal annealing step in vacuum was necessary to get phasepure products. In 2015, using an electric ball-mill Prochowicz et al. demonstrated phase-pure $\mathrm{MAPbI}_{3}$ without the need of any further treatment. ${ }^{[20]}$ Since then, several tens of articles have been published reporting the MCS of LHPs and related materials, which indicates a growing interest on this synthetic method for such semiconductors. 
In this manuscript, we will review the recently published manuscripts regarding the use of MSC for the preparation of LHPs and other multinary metal halides. Additionally, we will discuss how MCS can assist to advance the following four areas; (i) the production of high-purity LHPs as dry powders and colloidal dispersions; (ii) the investigation of fundamental properties of mixed-composition LHPs; (iii) the development of related multinary metal halides (lowdimensionality lead halides as well as lead-free ternary and quaternary alternatives); and (iv) the implementation of all these materials into optoelectronic devices by processing the assynthesized products into thin films. Below we will comment in detail on each of these four areas of research.

\section{1) MCS for the production of high-purity LHPs}

a. Dry / bulk

As mentioned in the introduction, LHPs have drawn considerable attention in the past decade as promising materials for optoelectronics. Conventionally, most LHPs are synthesized by solution processing. More specifically, halide salts are commonly dissolved in organic solvents (dimethyl formamide -DMF- and dimethyl sulfoxide -DMSO- are the most frequent choices) and used to form perovskite thin films by spin-coating either in a single step or using one of many sequential processes. While this approach has proven to yield excellent results in a rather simple manner, it has a few drawbacks and limitations. These are, the limited choice of solvents for the LHP metal precursor salts, the toxicity of some of the solvents, challenging upscaling, and limitations in composition freedom and stoichiometry due to the need of a good common solvent for all precursor salts. In contrast, MCS is solvent free, easily upscalable, can lead to high purity materials and it has virtually no limitations in composition or stoichiometry. Furthermore, it has been shown that MCS-LHPs show superior stability for long periods of time. ${ }^{[21,22]}$ Therefore MCS represents a very appealing synthetic method not only from a scientific point of view but also from a technological and cost-analysis point of view. 
$\mathrm{APbX}_{3}$ LHPs can be synthesized by adding stoichiometric amounts of the binary constituents ( $\mathrm{AX}$ and $\mathrm{PbX}_{2}$ ) in a mortar and grinding by hand with a pestle (see Figure 1a). This simple method was first demonstrated in 2013 by Stoumpos et al. who synthesized different hybrid organic-inorganic iodide perovskites in this way. ${ }^{[19]}$ The same hand grinding approach was later employed by several other groups to prepare similar LHPs. ${ }^{[19,23-30]}$ While this is probably the simplest possible approach, hand grinding can be tedious and most importantly may lead to unreacted precursor impurities in the sample. Hence, electrically-powered ball-milling is now used predominantly as it is a more convenient and widely-used technique for MCS (see Figure 1b). ${ }^{[13,20,22,24,26-28,31-52]}$ In this approach, precursors are loaded into a sealed milling jar together with several beads that act as grinding medium. The sealing is generally airtight which allows for the operation in inert atmosphere if the jars are loaded and unloaded in an inert glovebox. The beads and the jars' inside walls are typically made out of a high-hardness material such as zirconia, corundum or agate, although stainless steel is also used. As the jar is set in motion (either by simple straight shaking or more commonly by rotating in so-called planetary instruments) mechanical energy (e.g., by compression, shear or friction) is translated from the grinding medium to the reaction mixture. This then leads to conversion of the precursors into the stable perovskite phase (see Figure 1b). As mentioned before, the completion of the synthesis occurs as upon milling fresh reactant surface is constantly exposed. ${ }^{[37,47,53-57]}$ MCS is especially promising for industrial upscaling. An interesting example of this was recently shown by Hong et al. who reported the synthesis of metal halide perovskites by ballmilling on the kilogram scale (see Figure 1c). ${ }^{[58]}$ MCS is already used in industry for several applications such as mechanical alloying or pharmaceutical drug synthesis on a larger scale. ${ }^{\text {[59] }}$ In recent years, all the pure 3D lead halide perovskites have been prepared by MCS with excellent phase purity, as determined by XRD (see Table 1). Additionally, a wide range of mixed compositions (see section 2) and different crystalline structures (see section 3) have also been reported. These achievements prove the high versatility of this simple preparation method. 
Several parameters can be tuned during ball-milling that affect the properties of the product. ${ }^{[60,61]}$ These include the milling time, the rotation or shaking frequency, and the ball-topowder weight ratio (common values are in the order of $10: 1) .{ }^{[58,61-65]}$ Recently, we have conducted a detailed investigation regarding the ball-milling MCS of $\mathrm{CsPbBr}_{3}$ which can be considered the archetype inorganic halide perovskite. ${ }^{[66]}$ Using fixed conditions (ball-topowder ratio and frequency), three different stages of reaction process could be observed: (i) In the first stage, the binary precursors reacted to form different ternary compounds including the stoichiometric $\mathrm{CsPbBr}_{3}$ and the non-stoichiometric $\mathrm{CsPb}_{2} \mathrm{Br}_{5}$ and $\mathrm{Cs}_{4} \mathrm{PbBr}_{6}$ compounds. (ii) In the second stage after approximately 5 minutes all these compounds reacted to form only the phase-pure $\mathrm{CsPbBr}_{3}$ that exhibited an intense and narrow photoluminescence at $525 \mathrm{~nm}$. (iii) In the third stage, further milling leads to the reduction of the crystal size and a loss of photoluminescence while preserving the phase purity. These results highlight that an optimum time exists (in this case rather short) to achieve the complete conversion before non-desired side reactions, or degradation products are formed that lead to detrimental optical properties.

\section{b. Colloidal QDs}

In the previous section, we have shown that ball-milling can lead to the synthesis of a wide range of LHPs (see Table 1). However, grinding of materials is most commonly used to reduce the size of the powder or grains of a material into finer particles and it is not generally associated with chemical changes in the grounded materials. Colloidal nanocrystals (NCs) and more specifically semiconductor quantum dots (QDs) have interesting optoelectronic properties beyond those of their bulk counterparts. Some reports describe the use of grinding to produce

CdSe and CdTe QDs. ${ }^{[11,12]}$ Nevertheless, this method has not been widely adopted, most likely, because grinding usually leads to a high number of crystal defects which is detrimental for most semiconductors as explained in the introduction. Because of their crystal defect-tolerant nature, halide perovskite QDs can be prepared by grinding while maintaining good optical 
properties. ${ }^{[29,47]}$ In some cases, LHP NCs with photoluminescence quantum yields in excess of $90 \%$ were obtained, demonstrating the excellent quality of these materials. This opens a new avenue, or rather enables an old one, for mass production of bright QDs. Two different strategies can be taken for the synthesis of halide perovskite QDs by MCS. The first approach consists of two steps: (i) synthesis of bulk compound by dry MCS as previously detailed or by any other method (e.g., solution synthesis of bulk single crystals), followed by (ii) reduction in size by MCS, usually in the presence of a solvent and surface-capping ligand molecules, following the reaction shown below:

$$
\begin{aligned}
& \text { (i) } A(s)+B(s) \stackrel{M C S}{\longrightarrow} A B(\text { bulk }) \\
& \text { (ii) } \quad A B(\text { bulk })+\text { solvent }+ \text { ligands } \stackrel{M C S}{\longrightarrow} A B(\text { colloidal })
\end{aligned}
$$

The second approach consists in the direct synthesis of the desired compound in presence of solvent and ligands, which can be written as depicted in the following formula:

$$
A(s)+B(s)+\text { solvent }+ \text { ligands } \stackrel{M C S}{\longrightarrow} A B(\text { colloidal })
$$

In 2016, Hintermayr et al. synthesized hybrid organic-inorganic $\mathrm{MAPbX}_{3}(\mathrm{X}=\mathrm{I}, \mathrm{Br}, \mathrm{Cl}$, and mixtures thereof) by the two-step approach. ${ }^{[25]}$ They first prepared $\mathrm{MAPbX}_{3}$ bulk crystals by grinding with mortar and pestle for 5 minutes followed by tip sonication in toluene in the presence of oleylamine (OlAm) ligands for 30 minutes. To the best of our knowledge this is the first report of lead halide perovskite NCs prepared by MCS. In fact, the NCs themselves are not formed by grinding as discussed so far though grinding is used as a first step to form bulk compounds. Furthermore, sonication can be seen as a related technique which also falls in the category of mechanochemistry. ${ }^{[67]}$ Upon this treatment, micrometric "bulk” perovskite crystals were exfoliated into thin nanoplatelets (see Figure 2a-b).

As can be seen in Figure 2b, most of the NCs are obtained in the form of 2D nanoplatelets or nanosheets. The authors mention that a small fraction of the NCs had different morphologies (cubes and rods). It is known that many morphologies can be obtained for halide perovskite 
QDs. The reason behind the predominance of the 2D structures for this particular case is not clear although it may be linked to the use of OlAm as only ligand. ${ }^{[68]}$ Interestingly, the thickness of the nanoplatelets could be reduced down to a single monolayer by selective centrifugation/purification steps. As a result of this fine composition control, the emission wavelength was altered due to the thickness-dependent quantum confinement effect (blue-shift). The authors described a wide range of compositions, from the pure $\mathrm{Cl}, \mathrm{Br}$ and I to mixed LHPs. This wide range of compositions enables light emission over the whole range of visible wavelengths (see Figure 2c). For one composition a maximum PLQY of 70\% was achieved, demonstrating that this method leads to good optical quality materials (the exact composition for which this value is obtained is not specified). Zhu et al. followed a similar two-step approach for the synthesis of fully inorganic $\mathrm{CsPbX}_{3}$ NCs. ${ }^{[4]}$ Here, also in a first dry ball-milling step the desired perovskite is formed. This as-prepared powder exhibited poor photoluminescence efficiency. Upon addition of a small amount of oleylamine (without any other solvent) and continued milling, nanocrystals were formed with efficient photoluminescence emitting over the whole visible wavelength range (see Figure 2d for PLQY and FWHM as a function of PL maximum). It is worth noting that in some cases, depending on the ligands and the milling time, Zhu et al. observed a crystal phase change. ${ }^{[4]}$ The reason behind this was not further commented upon in the manuscript. Based on the rhombohedral shapes observed by TEM and more importantly on the XRD signal especially at long milling times, we infer that $\mathrm{Cs}_{4} \mathrm{PbBr}_{6}$ is formed under certain conditions. This is plausible, as it is known that oleylamine can induce the transformation of preformed $\mathrm{CsPbBr}_{3}$ into $\mathrm{Cs}_{4} \mathrm{PbBr}_{6}{ }^{[68,69]}$ Nevertheless this highlights an important point for the two-step approach of making perovskite QDs, which is that addition of ligands (necessary for colloidal stability) may also result in unwanted further chemical reactions. A second method in which the colloidal synthesis is achieved by grinding the binary halide precursors directly in wet conditions, in the presence of solvent and ligands has also been reported. This is also referred to as liquid-assisted grinding (LAG) ${ }^{[10]}$ Wet MCS of LHPs has 
been done by manual and electrically-powered ball milling under continuous addition of cyclohexane, ${ }^{[29]}$ and toluene, ${ }^{[70]}$ respectively, as well as in solvent-free conditions with only octylammonium bromide ligands. ${ }^{[71]}$ Variations of the method have been reported in which different solvents were used to disperse the NCs, such as mesitylene, octadecene, diphyl, hexane, and chloroform. ${ }^{[13]}$ Protesescu et al. found mesitylene to be the best solvent with respect to colloidal stability. ${ }^{[13]}$ They also found that using oleylammonium halide as a ligand instead of the more classical combination of oleylamine and oleic acid leads to more monodisperse NCs yielding narrower PL linewidths. As in the case of the two-step method, many different halide perovskite NCs have been synthesized with emission wavelengths spanning the whole visible spectrum. In particular, Chen et al. recently reported the synthesis of Cs-, MA-, and FAbased lead halide perovskite NCs with $\mathrm{X}=\mathrm{I}, \mathrm{Br}$, and $\mathrm{Cl}$ and some mixed compositions. ${ }^{[29]} \mathrm{A}$ maximum PLQY of $92 \%$ was achieved for MAPbBr3 with an emission centered at $525 \mathrm{~nm}$ and a FWHM of $25 \mathrm{~nm}$. This is very similar to what has been reported for NCs with the same composition prepared using solution based colloidal syntheses. In spite of these promising achievements, several limitations or side-effects of MCS based preparation have been pointed out by different groups. Though no spectral changes were observed, Chen et al. noted a decrease of the PLQY with prolonged grinding times above 30 min, and especially beyond 2 hours. ${ }^{\text {[29] }}$ During the synthesis of $\mathrm{FAPbBr}_{3} \mathrm{NCs}$, Protesescu et al. noted important spectral differences with prolonged milling. ${ }^{[13]}$ The main emission peak of $\mathrm{FAPbBr}_{3}$ centered at $537 \mathrm{~nm}$ obtained after $1 \mathrm{~h}$ of milling was found to blue-shift to $450 \mathrm{~nm}$ after $3 \mathrm{~h}$ probably due to the generation of different quantum-confined morphologies. This is in agreement with reports from Yun et al. who also found different morphologies in the synthesis of $\mathrm{FAPbBr}_{3}{ }^{[70]}$ While the loss of PLQY in these wider bandgap perovskites may be ascribed to formation of deep traps linked to crystal defects, the blue-shift is most probably due to a reduction of NC size into quantum-confined nanoplatelets. According to Protesecu et al., the PL spectra are also narrower when the twostep method is used starting from bulk single perovskite crystals than when direct synthesis is 
attempted $(\mathrm{FWHM}=27 \mathrm{~nm}$ vs $49 \mathrm{~nm}$ respectively for $\mathrm{CsPbBr} 3) .{ }^{[13]}$ This is due to the fact that when direct synthesis is carried out, the nucleation of the perovskite phase will start at different times for different fractions of the sample, leading to a larger poly-dispersion at the end of the reaction. Mixed-halide NC compositions have also been prepared using MSC. Chen et al. noted that in the case of $\mathrm{FAPb}(\mathrm{Br}: \mathrm{Cl})_{3}$, pure-bromide $\mathrm{FAPbBr}_{3}$ seemed to form preferentially at shorter grinding times, with the subsequent anion-exchange with chloride only at longer times. ${ }^{[29]}$ Protesescu et al. also synthesized mixed-halide FAPb(Br:I) 3 NCs by first synthesizing the pure-bromide counterpart and performing anion-exchange in a following step with oleylammonium iodide and obtained better results than by direct synthesis of the mixed composition. ${ }^{[13]}$ The authors mentioned that their method was not applicable to pure iodide compositions.

It is also worth noting that halide perovskite NCs employing different A-type monocations have been achieved by direct MCS. Yun et al. synthesized mixed-cation lead bromide perovskites by partial substitution of MA by FA or ethylammonium (EA). ${ }^{[70]}$ The insertion of EA had a positive impact on the PLQY, raising the value from $20 \%$ to $80 \%$. We note however that even this “enhanced” PLQY is lower than obtained by Chen et al. and others for pure MAPbBr $3 .{ }^{[29]} \mathrm{MCS}$ has also been used to prepare $\mathrm{Mn}$-doped $\mathrm{CsPbCl}_{3}$ by addition of $\mathrm{MnCl}_{2}$ to the other precursors. [29] This leads to a material with a large Stokes-shift between the absorbance occurring mainly in the $\mathrm{CsPbCl}_{3}$ matrix and the emission originating from the $\mathrm{Mn}^{2+}$ dopants. According to the authors such a material is interesting for luminescent solar concentrators. ${ }^{[72]}$ In a simpler application, Chen et al. embedded red-, green- and blue- emitting QDs prepared by MCS in PMMA to form stacked color-converting layers on top of a UV LED to obtain a white light (CIE coordinates $(0.360,0.354))$ with a color rendering index (CRI) of 85. ${ }^{\text {[29] }}$

In conclusion, large-scale synthesis of lead halide perovskite colloidal QDs can be achieved either by direct wet grinding of precursors or by size reduction of pre-formed bulk perovskites. Both approaches have been implemented for a wide range of compositions, including fully 
inorganic $\mathrm{CsPbX}_{3}$ as well as hybrid organic-inorganic $\mathrm{MAPbX}_{3}$ and $\mathrm{FAPbX}_{3}$, where $\mathrm{X}$ is $\mathrm{Cl}$, Br, I, or mixtures thereof. Furthermore, PLQY values higher than 90\% are reported. Hence, in many aspects the resulting material can be considered on par to those obtained by more complex traditional colloidal syntheses. Nevertheless, we observe that obtaining good size and shape monodispersity is still challenging by MCS. As the number of reports on this is still relatively low, it is not possible to determine whether this limitation is intrinsic to the technique or is linked to the lack of standard parameters (such as grinding time, ball-to-material weight ratio for milling, appropriate solvents and ligands) that may be developed as MCS becomes more widely implemented by the community working on halide perovskite QDs.

\section{2) MCS as a way to investigate mixed-composition halide perovskites}

Halide perovskites, as already discussed, can be simply written as $\mathrm{ABX}_{3}$ compounds. However, this simple formula somehow hides one fundamental aspect that has motivated a lot of research on these materials, which is that several "A", "B", and " $\mathrm{X}$ ” elements can be combined in a single, homogeneous, perovskite structure. This high degree of tolerance towards mixedcompositions is not only interesting from a fundamental chemistry point of view, but it also provides a wide range of possibilities to fine-tune the optoelectronic properties of the final material for different applications. A very clear example of this can be found by observing that some of the best performances in perovskite photovoltaics to date have been obtained with rather complex formulations such as (Rb:Cs:MA:FA)Pb(I:Br) $3 .{ }^{[73]}$ Indeed, mixing different elements in the A, B, and/or X-sites of halide perovskites may result in several physical and chemical changes (see Figure 3).

In a very simple summary of the main effects encountered so far we may highlight the following: 
- A-site mixing has been found particularly fruitful to stabilize the perovskite phase by tuning the Goldschmidt tolerance factor, defined as $t=\frac{r_{A}+r_{X}}{\sqrt{2}\left(r_{B}+r_{X}\right)}$; where $\mathrm{r}_{\mathrm{A}}$, $\mathrm{r}_{\mathrm{B}}$, and $\mathrm{r}_{\mathrm{X}}$ are the effective ionic radiuses of $\mathrm{A}, \mathrm{B}$, and $\mathrm{X}$. It has been found that to obtain a stable photoactive perovskite structure, t should have a value between 0.9 (or 0.8 ) and 1. Figure 3a shows the tolerance factor of LHPs as a function of $r_{A}$ (ionic radiuses for different reported atoms and molecules are obtained from Shannon et al. ${ }^{[74]}$ and Kieslich et al. ${ }^{[75]}$ ). As can be seen MA-based lead trihalides have an ideal tolerance factor (between 0.9 and 1), whereas Cs-based and FA-based ones are at the lower and higher boundaries of 0.8 and 1 . Indeed, $\mathrm{CsPbI}_{3}$ and $\mathrm{FAPbI}_{3}$ are reported to be thermodynamically unstable in the cubic perovskite phase at room temperature. Nevertheless, mixed $\left(\mathrm{Cs}_{\mathrm{x}} \mathrm{FA}_{1-\mathrm{x}}\right) \mathrm{PbI}_{3}$ can crystallize in a perovskite structure at room temperature in a wide range of $\mathrm{x}$ values. ${ }^{[76]}$

- $\quad$ B-site is occupied by $\mathrm{Pb}^{2+}$ cations in most cases. Nevertheless, mixing $\mathrm{Pb}$ (II) and $\mathrm{Sn}(\mathrm{II})$ is a promising strategy for low bandgap halide perovskites, as different $\mathrm{A}(\mathrm{Pb}: \mathrm{Sn}) \mathrm{X}_{3}$ perovskites have been found to have a lower bandgap than both $\mathrm{APbX}_{3}$ and $\mathrm{ASnX}_{3}$ (see Figure 3b). B-site doping is also being actively investigated for other purposes such as to decouple absorption and emission properties (Figure 3c).

- X-site mixing has been extensively demonstrated to have a large effect on the bandgap. Indeed, mixed-halide $\mathrm{APb}(\mathrm{Br}: \mathrm{I})_{3}$ and $\mathrm{APb}(\mathrm{Br}: \mathrm{Cl})_{3}$ can be obtained with bandgaps spanning the whole visible range just by changing the relative concentrations of different anions (Figure 3d).

This demonstrates that mixing different elements in a single halide perovskite material is not only possible, but represents an interesting route to fine-tune the materials’ properties. Hence, a lot of research efforts are devoted to investigate mixed-composition halide perovskites. In order to carry on these investigations, it is very important to have synthetic routes where the 
different elements can be added with a precise control, without the interference of additives (e.g., solvents or ligands) or having to deal with solubility differences and limitations. Furthermore, as the properties of halide perovskites can be greatly influenced by the substrate on which these are deposited or grown, it is also important to synthesize them in a nontemplated manner, to ensure that the observed properties are inherent to the material itself. MCS appears thus as an ideal synthetic method to fulfill all these requirements. This is evidenced by the multitude of recent studies describing the MSC preparation of LHPs with different A-, B-, and X-site compositions. [13,24,25,27-29,31,34,36,38,39,41-43,45,47,70,77,78]

\section{A. A-site mixing}

Initial reports on organometal halide perovskites for photovoltaics concentrated on $\mathrm{MAPbI}_{3}$ (MAPI). While this material indeed shows excellent photovoltaic properties and is still widely used today, its significant degradation upon heating and its rather wide bandgap for single junction solar cells make it non-ideal for photovoltaics. $\mathrm{FAPbI}_{3}$ (FAPI) instead, possesses a slightly lower bandgap, improving the absorption in the near infrared part of the solar spectrum and also shows enhanced thermal stability. Hence, in recent years FAPI has been more actively investigated for high-performance solar cells. Unfortunately, FAPI crystallizes in the so-called “yellow” orthorhombic non-perovskite phase ( $\delta$-FAPI) at room temperature. This can be linked to its large Goldschmidt tolerance factor (see Figure 3a). In order to tune this geometric factor and ensure a stable cubic perovskite phase at room temperature, a common strategy is to introduce smaller A-cations into FAPI as previously mentioned. The main question that arises from this is what is the minimum amount of smaller A-cations that must be introduced in the lattice to maintain as much as possible the beneficial properties of FAPI while ensuring a cubic phase at room temperature. This is a question that can be conveniently investigated by MCS where it is easy to mix different precursors in very accurate ratios. Prochowicz et al. synthesized mixed-cation $(\mathrm{MA})_{\mathrm{x}}(\mathrm{FA})_{1-\mathrm{x}} \mathrm{PbI}_{3}$ by MCS with $\mathrm{x}=0.15,0.20$, and $0.25 .{ }^{[45]}$ This is to the best of 
our knowledge the first report on mixed-cation halide perovskites prepared by MCS. The authors found that a fraction of $\delta$-FAPI was noticeable by XRD for $\mathrm{x}=15 \%$ and $\mathrm{x}=20 \%$ but vanished for compositions with MA above 25\%. These mixed-perovskites were later used for the formation of thin film absorbers in solar cells, as will be detailed in the last part of this review. In a following paper, the same group used similar materials for a fundamental investigation of cation dynamics by solid-state NMR. ${ }^{[49]}$ Their study showed that FA, though bulkier than MA, exhibits faster reorientation dynamics and longer carrier lifetimes. The same reasoning that has been made for the stabilization of FAPI through insertion of MA can be made by replacing MA with $\mathrm{Cs}^{+}$which has an even smaller ionic radius. Furthermore, cesium-based perovskites have an increased thermal stability. Kubicki et al. demonstrated homogeneous mixed-cation $(\mathrm{Cs})_{\mathrm{x}}(\mathrm{FA})_{1-\mathrm{x}} \mathrm{PbI}_{3}$ by MCS with a cesium loading up to around $15 \% .{ }^{[77]}$ Recently, Prochowicz et al. repeated this synthesis for another study. ${ }^{[43]}$ What is interesting to note here, is that this report focusses not on MCS but on films made fully by solution processes. However, in order to corroborate an important hypothesis on the process (namely the formation and subsequent sublimation of FACl upon post-synthesis annealing), the authors prepare freestanding bulk analogue powders by MCS. These powders were characterized using infrared spectroscopy, mass spectrometry, and powder XRD. Hence, here MCS was used to enable analysis of the pure materials (rather than films which are always on a surface) and which can help to elucidate the more fundamental properties of complex perovskites.

Beyond the three most commonly employed A-site cations in 3D halide perovskites, Cs, MA, and FA, a growing interest is being developed on the possible roles of even smaller inorganic cations such as $\mathrm{Rb}^{+}$and $\mathrm{K}^{+}$. Both of these have been investigated by MCS. ${ }^{[31,34,77]}$ Kubicki et al. demonstrated that, in the case of iodide 3D perovskites, neither $\mathrm{Rb}^{+}$nor $\mathrm{K}^{+}$were effectively incorporated in the perovskite matrix at the atomic level. ${ }^{[77]}$ In contrast, segregated $\mathrm{RbPbI}_{3}$ domains were found in all Rb-doped samples whereas in the case of $\mathrm{K}^{+}$simply unreacted $\mathrm{KI}$ was found. It is worth noting that the authors "address the question of whether bulk 
mechanochemically synthesized perovskites are a good representation of the thin films used in PV devices. Comparison of NMR spectra between a bulk mechanochemical triple-cation Cs/MA/FA perovskite and a thin film prepared by spin-coating shows no significant differences between the two materials, validating that bulk mechanochemical perovskites can be used to obtain structural information about newly developed [halide perovskites] systems. "[77] In a follow-up paper, the same authors provided further evidence of phase segregation in potassiumdoped pure iodide or iodide-rich perovskites. ${ }^{[34]}$ In the case of bromide perovskites, because of the smaller ionic radius of $\mathrm{Br}^{-}$in regards to $\mathrm{I}^{-}$, the tolerance factor is generally larger and smaller A-cations can be considered. Hence, we tested the incorporation of different potassium halide salts in the mechanochemical synthesis of $\mathrm{CsPbBr}_{3} .{ }^{[31]}$ We found that a small fraction of $\mathrm{K}^{+}$ could effectively be incorporated in the 3D perovskite matrix. Nevertheless, we also observed the formation of $\mathrm{KPb}_{2} \mathrm{Br}_{5}$ as a side-product with $20 \%$ loading of potassium. As longer PL decay times were observed upon potassium incorporation, we speculate that this nonperovskite phase can act as a passivating surface layer, in accordance with other reports. ${ }^{[79]}$

Larger organic cations than FA have also been considered, such as guanidinium $(\mathrm{GuA})^{[24]}$ and ethylammonium (EA). ${ }^{[70]}$ Although these are too large to be considered as the only A-cations in 3D halide perovskites, they have been introduced by MCS in mixed-cation 3D perovskites. Kubicki et al. ${ }^{[24]}$ incorporated different fractions of guanidinium in MAPI and FAPI. As expected, the cubic phase of the mixed (GuA:FA)PbI 3 compounds were found to be unstable since both A cations give excessively high tolerance factors. However, mixed $\mathrm{GUA}_{\mathrm{x}} \mathrm{MA}_{1-\mathrm{x}} \mathrm{PbI}_{3}$ compositions with $\mathrm{x}<0.4$ were found to be stable for over a month. Moreover, the authors found that these compositions showed longer charge carrier lifetimes and improved photovoltaic performances as compared to pure MAPI. Yun et al. synthesized (EA)x(MA)1${ }_{x} \mathrm{PbBr}_{3}$ nanocrystals by wet MCS and found a very significant and rather linear increase of the PLQY from $20 \%$ for $\mathrm{x}=0$ to $80 \%$ for $\mathrm{x}=10 .{ }^{[70]}$ Nevertheless these values remain below the 
highest PLQY values achieved for neat $\mathrm{MAPbBr}_{3} \mathrm{NCs}$ synthesized by MCS, as discussed in the previous section.

\section{B. B-site mixing}

While most halide perovskites use $\mathrm{Pb}(\mathrm{II})$ as unique B-cation, the partial or total replacement of Pb(II) by Sn(II) has also been investigated by MCS. ${ }^{[19,41,58,80]}$ Saski et al. reported pure-tin mixed-halide perovskites -ASn(Br:I) ${ }_{3}$ - with good phase-purity under inert atmosphere. ${ }^{[41]}$ Oxidation of Sn(II) to Sn(IV) in air was found to be suppressed by addition of a small amount of $\mathrm{SnF}_{2}$ during MCS. Hong et al. extended the range of explored pure-tin(II) compounds to chlorides and mixed bromide-chloride compositions. ${ }^{[58]}$ We recently demonstrated the synthesis of several mixed lead-tin and pure tin perovskites iodide perovskites as well as socalled "vacancy-ordered perovskites" (based on Sn(IV), see next section for more details) and studied their thermal stability as well as their optical and electrical characteristics to assess their applicability in photovoltaics and NIR LEDs. ${ }^{[80]}$

Other examples of B-site mixing by MCS include the doping of lead halide perovskites with $\mathrm{Co}^{2+}$ or $\mathrm{Mn}^{2+} \cdot{ }^{[29,42]}$ Kubicki et al. showed different behaviours of these paramagnetic dopants: while $\mathrm{Co}^{2+}$ incorporation into MAPI led to phase segregation in all cases, they showed that $\mathrm{Mn}^{2+}$ can readily be incorporated in the crystal lattice of inorganic $\mathrm{CsPbCl}_{3}$ and $\mathrm{CsPbBr}_{3}$ perovskites up to $3 \%$ and $8 \%$ respectively. ${ }^{[42]}$ This approach was used by Chen et al. to synthesize $\mathrm{Mn}$-doped $\mathrm{CsPbCl}_{3}$ colloidal quantum dots, with a broad main photoluminescence peak around $600 \mathrm{~nm}$ corresponding to the $\mathrm{Mn}^{2+}{ }^{4} \mathrm{~T}_{1} \rightarrow{ }^{6} \mathrm{~A}_{1}$ transition largely Stokes-shifted from the absorption onset of the $\mathrm{CsPbCl}_{3}$ host structure. ${ }^{[81]}$ Such materials are promising for use in luminescent solar concentrators. ${ }^{[72]}$ Eu-doping has also been investigated on fully-inorganic perovskites by Xiang et al. ${ }^{[82]}$ The authors found that the incorporation of $5 \%$ europium (Eu(II) or $\mathrm{Eu(III))} \mathrm{led} \mathrm{to} \mathrm{improved} \mathrm{open-circuit} \mathrm{voltages} \mathrm{in} \mathrm{solar} \mathrm{cells} \mathrm{by} \mathrm{reducing} \mathrm{non-radiative}$ recombination. Here, somewhat surprising is that both the bi- and tri-valent Eu ions lead to 
similar effects. This would indicate that the effect is not primarily related to lattice incorporation of the Eu ions. Doping with different rare earth elements such as $\mathrm{Yb}^{3+}$ has also been recently demonstrated by MCS. ${ }^{[83]}$ These $\mathrm{Yb}^{3+}: \mathrm{CsPb}(\mathrm{Cl}: \mathrm{Br})_{3}$ (5\% doping) results in quantum-cutting, that is the emission of more than one infrared photon by the $\mathrm{Yb}^{3+}{ }^{2} \mathrm{~F}_{5 / 2} \rightarrow{ }^{2} \mathrm{~F}_{7 / 2}$ transition for each visible or UV photon absorbed by the perovskite, resulting thus into a PLQY higher than unity. This is, to the best of our knowledge, the first report on quantum-cutting perovskites prepared by MCS.

\section{X-site mixing}

Most mixed-composition halide perovskites are based on combining different halide anions. Indeed, out of the three elements present in the $\mathrm{ABX}_{3}$ structure, a change in $\mathrm{X}(\mathrm{X}=\mathrm{I}, \mathrm{Br}, \mathrm{Cl}$ or mixtures thereof) is usually the most effective way of tuning the optical bandgap (see Figure 1d). As we've previously seen, this has been used to make brightly emissive quantum dots over the whole visible range (see Figure 2). ${ }^{[13,25,47,81]}$ Mixed-halide bulk perovskites have also been investigated by MCS. ${ }^{[27,28,30,31,35,41,48,58,78,82]}$ Generally, it is found that $\mathrm{Br}-\mathrm{I}$ and $\mathrm{Br}-\mathrm{Cl}$ mixed compositions can be formed with any ratio of both halides. This can be achieved either by mixing the halide precursors or by grinding the preformed single-halide perovskites in the desired ratios. For example, Karmakar et al. prepared $\mathrm{MAPb}\left(\mathrm{Br}_{\mathrm{x}} \mathrm{Cl}_{1-\mathrm{x}}\right)_{3}$ perovskites by hand grinding and ball-milling of pre-synthesized $\mathrm{MAPbBr} 3$ and $\mathrm{MAPbCl}_{3 .}{ }^{[28]}$ Compared to solution processing, the authors note that: "The solvent-free mechanochemical synthesis approach [reveals] identical solid-solution behavior; however, the mechanochemical approach offers superior control over the stoichiometry of the final mixed-halide composition, which is essential for device engineering." Extensive NMR characterization showed the perfect mixing of halides in a single crystalline phase without any amorphous or phase segregated domains. 
Aside from incorporating different A-, B-, and X-elements, MCS can be used to test the addition of different molecules that may act as passivating agents by removing surface trap states. This is a topic of high importance in the development of halide perovskites as the presence of potential trap states directly influences the device properties when used in solar cells or LEDs. ${ }^{[79,84]}$ In this context, Tavakoli et al. prepared adamantylammonium-passivated FAPI by ball-milling ADAHI:FAI:PbI 2 in a 2:29:30 ratio (ADAHI = adamantylammonium iodide). ${ }^{[36]}$ By detailed solid-state NMR, the authors are able to evidence the interaction of ADAHI dopant and FA "on the microscopic scale, consistent with a surface-type interaction”. Many other species have been proposed for surface passivation of halide perovskites, such as phosphine oxides. ${ }^{[84]}$ We believe that MCS could be an ideal platform to test these in a systematic and robust way.

\section{3) MCS to explore other multinary metal halides}

a) 2D and quasi-2D Ruddlesden-Popper structures

Hybrid organic-inorganic Ruddlesden-Popper lead halides are typically obtained by using longchain (LA) and short-chain (SA) ammonium cations. ${ }^{3}$ In doing so, a layered structure is obtained combining "slabs" of various thicknesses of 3D perovskites separated by long amines, with the general formula $(\mathrm{LA})_{2}(\mathrm{SA})_{\mathrm{n}-1} \mathrm{~Pb}_{n} \mathrm{X}_{3 \mathrm{n}+1}$, where $\mathrm{n}$ is the number of monolayers of $3 \mathrm{D}$ perovskites in the slabs. ${ }^{[37,44,85-88]}$ These materials have recently triggered a lot of interest, among other reasons due to their increased stability with respect to the 3D counterparts. ${ }^{[89]}$ Posudievsky et al. have used MCS to develop 2D crystals of hexylammonium lead iodide $\left(\left(\mathrm{C}_{6} \mathrm{H}_{13} \mathrm{NH}_{3}\right)_{2} \mathrm{PbX}_{2}\right)$ with narrow visible photoluminescence. ${ }^{[32]}$ Wilke et al. ${ }^{[37]}$ and Jodlowski et al. ${ }^{[44]}$ have mechanochemically synthesized hybrid organic-inorganic 2D guanidinium lead iodides $\left(\left(\mathrm{C}\left(\mathrm{NH}_{2}\right)_{3}\right)_{2} \mathrm{PbI}_{2}\right)$ as well as other guanidinium-based structures. It is worth highlighting

\footnotetext{
${ }^{3}$ Note that also fully inorganic Ruddlesden-Popper structures exist, such as $\mathrm{Cs}_{2} \mathrm{PbCl}_{2} \mathrm{I}_{2}$.
} 
that Wilke et al. performed in-situ XRD characterization to show that full conversion takes place in only about 20 minutes. ${ }^{[37]}$ This reaction time is similar to what we have found on 3D LHPs. $^{[66]}$ Eventually $\mathrm{Li}$ et al. used dry grinding to synthesize a series of $\left(\mathrm{NH}_{3} \mathrm{C}_{\mathrm{m}} \mathrm{H}_{2 \mathrm{~m}} \mathrm{NH}_{3}\right)\left(\mathrm{CH}_{3} \mathrm{NH}_{3}\right)_{\mathrm{n}-1} \mathrm{~Pb}_{\mathrm{n}} \mathrm{I} 3_{\mathrm{n}+1}$ compounds with $\mathrm{m}=4-9$ and $\mathrm{n}=1-4$. The number of inorganic layers in the slabs (n) mostly determined the bandgap of the material, ranging from $2.58 \mathrm{eV}(\mathrm{n}=1)$ to $1.89 \mathrm{eV}(\mathrm{n}=4)$, while an increasing number of carbons in the alkyl chains (m) resulted in a preferential orientation of thin films cast on glass substrates. ${ }^{[90]}$

b) Other ternary cesium lead halides

$\mathrm{CsPbX}_{3}$ are well-known inorganic perovskites, of which several reports by MCS exist (see section 1). Nevertheless, this is not the only stable ternary cesium lead halide phase. Two other examples, namely $\mathrm{CsPb}_{2} \mathrm{X}_{5}$ and $\mathrm{Cs}_{4} \mathrm{PbX}_{6}$ have also been reported, and are currently under intense investigation to elucidate their properties. ${ }^{4[26,40,78]}$ Karmakar et al. recently showed that by simply changing the starting ratio between $\mathrm{CsX}$ and $\mathrm{PbX}_{2}$ it is possible to either obtain $\mathrm{CsPbX}_{3}$ or $\mathrm{Cs}_{4} \mathrm{PbX}_{6}$ by hand grinding. ${ }^{[78]}$ However, they observed about 5\% of unidentified impurities in the case of $\mathrm{Cs}_{4} \mathrm{PbX}_{6}$. It is nevertheless possible that if more energetic and longer ball-milling was used instead of hand grinding with mortar and pestle, these impurities would vanish.

\section{c) Lead-free multinary metal halides}

Besides all of the above-mentioned ternary lead halide structures, a wide variety of multinary metal halides can be obtained by replacing divalent lead by one or several metallic cations of different valences (see Figure 4). This is highly sought after as $\mathrm{Pb}(\mathrm{II})$ is toxic. Nevertheless, it

\footnotetext{
${ }^{4}$ An important matter of current debate is the photoluminescence observed in some reports from $\mathrm{Cs}_{4} \mathrm{PbBr}_{6}$, which is attributed by some to $\mathrm{CsPbBr}_{3}$ impurities and by other to an intrinsic property of the material. This is however beyond the scope of the present review.
} 
is not easy to find common solvents for different metal halides, in view of their limited solubilities. ${ }^{[91]}$ Hence, fully-dry MCS is as a very promising approach to circumvent this issue and enable a broader screening of a multitude of related materials that are sometimes loosely referred to as "lead-free perovskites”. The reference to "perovskite”, however, is arbitrary and based on the crystal structure of many of these, as can be seen from Figure 4, even inappropriate. Breternitz et al. have recently reported the mechanochemical synthesis of $\mathrm{Cs}_{2} \mathrm{Ag}(\mathrm{I}) \mathrm{In}(\mathrm{III}) \mathrm{Br}_{6}$.

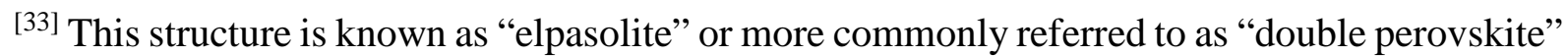
(Figure 4a). Double-perovskites, i.e., 3D crystals that have similar structure than standard perovskites with $\mathrm{B}(\mathrm{II})$ elements replaced by a combination of $\mathrm{B}(\mathrm{I})$ and $\mathrm{B}(\mathrm{III})$, are among the most-investigated lead-free multinary metal halides. However, as previously mentioned, solution-processing of these is limited by the poor solubility of the precursors. ${ }^{[91]}$ We can however expect that MCS will boost the development of these materials, as solubility does not represent a bottleneck in this approach. Other lead-free ternary metal halides can be formed with tetravalent tin (Figure 4b). We have recently demonstrated the mechanochemical synthesis of fully-inorganic $\mathrm{Cs}_{2} \mathrm{SnI}_{6}$ as well as hybrid organic-inorganic $\mathrm{FA}_{2} \mathrm{SnI}_{6 .}{ }^{[80]}$ As the tin is already in its highest +4 oxidation state, these compounds do not suffer from further oxidation as is the case with Sn(II)-based perovskites (see previous section 2b). If, instead of tetravalent cations, trivalent ones are used such as $\mathrm{Bi}(\mathrm{III})$, different $0 \mathrm{D}$ and 2D structures can be obtained with the chemical formula $\mathrm{A}_{3} \mathrm{~B}_{2} \mathrm{X}_{9}$ (Figure $4 \mathrm{c}$ ). We,$^{[52]}$ and others ${ }^{[38,92]}$ have recently demonstrated MCS of various hybrid organic-inorganic as well as full-inorganic bismuth-based $\mathrm{A}_{3} \mathrm{Bi}_{2} \mathrm{X}_{9}$ crystals with tunable bandgaps and photoluminescence in the visible range. Sarkar et al. observed a solvatochromic effect in the liquid-assisted grinding synthesis of $\mathrm{Cs}_{3} \mathrm{Bi}_{2} \mathrm{I}_{9}$, yielding a darker brown product when the synthesis was carried out in N-methyl-2-pyrrolidone (NMP) and brighter red in ethanol. ${ }^{[92]}$ The origin of this effect, however, is not fully clear. Eventually other lead-free multinary metal halides such as $\mathrm{Cs}_{4} \mathrm{CuSb}_{2} \mathrm{Cl}_{12}$ or $\left(\mathrm{Ag}_{1-\mathrm{x}} \mathrm{Cu}_{\mathrm{x}}\right)_{2} \mathrm{HgI}_{4}$ with interesting magnetic or thermochromic properties have also been demonstrated by MCS. ${ }^{\text {93,94] }}$ 


\section{4) Processing and applications of mechanochemically-synthesized materials}

As we have detailed so far, MCS is an ideal route to synthesize and study a broad range of multinary metal halides with interesting optoelectronic properties. One of the characteristics of the method is that the material is obtained in fine powder shape which has benefits and disadvantages. It allows for the direct characterization independent of interfaces or substrates, as we mentioned in the previous sections. However, to employ these materials in diode-based applications (LEDs, solar cells), these powders need to be deposited into thin films where light and/or electrical charges can be injected and extracted. Hereafter we will discuss different strategies that have been developed for the processing of MCS-materials and implementation in different devices (see Figure 5).

In a relatively simple approach, Chen et al. used mechanochemically-synthesized colloidal QDs blended in PMMA as down-conversion layers (see Figure 5a). ${ }^{[29]}$ Separate blue-, green- and red-emitting films of millimetric thickness and centimeter lateral size were prepared with excellent color purity. Three different MCS NC based $\mathrm{MAPbCl}_{1.5} \mathrm{Br}_{1.5}, \mathrm{MAPbBr}_{3}$, and $\mathrm{MAPbBr}_{2} \mathrm{I}$ were homogeneously dispersed in transparent PMMA. By stacking the three materials (with optimized thicknesses) on top of a commercial UV light-emitting diode, a nearideal white light with CIE color coordinates $(0.360 ; 0.354)$ and excellent color rendering index of 85 was achieved. Nevertheless, luminous efficiency remained rather low at a value of 15 $\operatorname{lm} / \mathrm{W}$, which can be partly ascribed to low PLQY of blue- and red-emitting NCs. In a similar application, we blended lead-free $\mathrm{Cs}_{3} \mathrm{Bi}_{2} \mathrm{X}_{9}(\mathrm{X}=\mathrm{Br}$ and I) with PMMA in fully-dry solid state and pressed the composite material into a homogeneous pellet. The resulting photoluminescence under UV excitation corresponded to a warm white light with correlated color temperature of $3615 \mathrm{~K}$. Pellets made from pressed as-synthesized perovskites were also used by Hong et al. in a slightly more sophisticated application as a visible photodetector (see Figure 5b). ${ }^{[58]}$ In this work, lead-free $\mathrm{CsSnBr}_{1.5} \mathrm{Cl}_{1.5}$ powders were pressed into a pellet on top 
of which gold electrodes were evaporated. Electrical current in the dark $\left(\mathrm{I}_{\text {off }}\right)$ and under $445 \mathrm{~nm}$ excitation of $693.1 \mathrm{~W} / \mathrm{m}^{2}$ ( $\left.\mathrm{I}_{\mathrm{on}}\right)$ was recorded showing a ratio $\mathrm{I}_{\mathrm{on}} / \mathrm{I}_{\text {off }}$ of about one order of magnitude, with an overall responsivity and detectivity of $8.1 \times 10^{-2} \mathrm{~mA} / \mathrm{W}$ and $1.9 \times 10^{-8} \mathrm{Jones}$, respectively. While these simple applications are interesting proofs of concept, more advanced applications such as light-emitting diodes and solar cells cannot be based on the active material being dispersed in an insulating matrix (Figure 5a) or simply pressed in macroscopic pellets (Figure 5b). For a more widespread implementation of MCS, the as-synthesized materials need to be processed into smooth homogeneous thin films, with typical thicknesses ranging from 10nm to 1 micron. Several published articles report on the dissolution of MCS-perovskites in organic solvents to be used as "inks" that can be spin-coated on a substrate to fabricate thin films, and devices thereof (Figure 5c). ${ }^{[20-22,32,35,45,50,95]}$ Prochowicz et al. fabricated MAPI thin films from presynthesized MAPI powders dissolved in DMSO and compared them to films prepared from a solution of $\mathrm{PbI}_{2}$ and MAI precursors, which can be considered a standard process. ${ }^{[95]}$ In both cases, film morphologies were very similar, without pinholes and with average grain sizes around 180nm - 200nm as observed by SEM. The film thicknesses could also be controlled by both approaches to be similar (200 nm). More importantly, films prepared from mechanochemically-synthesized MAPI could be implemented in a single junction solar cell with a power conversion efficiency (PCE) on par to that prepared from the standard solution process and with lower hysteresis attributed to a lower surface defect density. A similar comparison was made with mixed MA-FA films prepared by both methods, where the device prepared from MCS perovskites outperformed significantly the one made from standard solution process (PCEs of 14.98\% and 10.97\% respectively), especially with a high short circuit current of $23.7 \mathrm{~mA} / \mathrm{cm}^{2} .{ }^{[45]}$ In a following paper, the authors were able to fabricate cesium chloride-containing perovskites films by dissolving presynthesized perovskites in DMF and DMSO, whereas $\mathrm{CsCl}$ itself was found to be non-soluble in the same solvents. ${ }^{[35]}$ This highlights a clear advantage of using MCS-perovskites as precursors in solution processing 
rather than their binary salt constituents. Another crucial benefit from a technological point of view was highlighted by Dou et al. ${ }^{[22]}$ Indeed, the authors verified that "inks" made by dissolving previously-mechanochemically-synthesized perovskites in the above-mentioned solvents were chemically stable for long periods of time, whereas inks made by dissolving all the different salts separately (without previous MCS) underwent rapid degradation even when stored under nitrogen in dark. Recently, Leupold et al. confirmed the exceptional stability of MCS-based perovskites (in dry powder form) without significant degradation for over two and a half years. $^{[21]}$

Despite these positive results based on dissolution of MCS-perovskites, it may be argued that such solution-processing somehow defeats one of the purposes of dry MCS, which is avoiding organic solvents. Therefore, dry processing methods to convert the as-synthesized powders into thin films are highly sought after. We have demonstrated single-source vacuum deposition (SSVD) of fully-inorganic MCS-perovskites to fabricate thin films of identical composition and optical properties in a simple and rapid way (see Figure 5d). ${ }^{[51]}$ In that manuscript we showed that this process works especially well for $\mathrm{CsPbCl}_{3}$ without any further processing, whereas for $\mathrm{CsPbBr}_{3}$ a post-deposition thermal annealing step was required in order to achieve high phasepurity in the film. Recently, others have adopted SSVD to fabricate quantum-cutting $\mathrm{Yb}^{3+}: \mathrm{CsPb}\left(\mathrm{Cl}_{1-\mathrm{x}} \mathrm{Br}_{\mathrm{x}}\right)_{3}$ films on planar and textured substrates with $183 \% \mathrm{PLQY} .{ }^{[83]}$ One of the challenges of SSVD resides in controlling film thickness. Indeed, when co-evaporation of different constituents is used to form the desired material at the substrate, the evaporation rate of all precursors can be finely adjusted and as such, the final thickness is controlled by the (slow) evaporation rates and total process time. ${ }^{[17,18]}$ On the opposite, when SSVD is employed, evaporation must be as fast as possible to avoid the incongruent melting and evaporation of different species at different temperatures. As such, the film thickness could be controlled by the amount of precursor fed into the thermal source, which should then be totally evaporated. 
The relationship between film thickness and precursor amount however could be impacted by other processing parameters such as the distance between the source and the substrate.

\section{Conclusion and outlook:}

As we have shown throughout this short review, mechanochemical synthesis (MCS) is emerging as a common approach for the development of lead halide perovskites (LHPs) and other multinary metal halides including lead-free alternatives. Several examples show that grinding or milling the binary precursors leads to the formation of the desired ternary compounds. While only a few recent reports have focused on describing the process in detail, something that could be greatly enhanced by in-situ characterization methods, there is no doubt that MCS can yield high-quality materials in a relatively short time $(<1 \mathrm{~h}) .{ }^{[96,97]}$ Indeed, all standard LHPs have been obtained with excellent phase purity by MCS (see Table 1, section 1a). Colloidal quantum dots with high photoluminescence quantum yields throughout the whole visible spectrum have also been synthesized via MCS by several groups (section 1b). Owing to the numerous advantages over more common solution-based methods (in terms of simplicity and toxicity among others), we can expect that the use of MCS will become more and more widespread in the field of perovskite optoelectronics in the near future. Furthermore, we have seen that MCS is an ideal route to investigate mixed-composition LHPs (section 2). While several examples have been given on $\mathrm{A}$-, $\mathrm{B}$-, and $\mathrm{X}$-site alloying on $\mathrm{ABX}_{3}$ perovskites (section 2a-c), we expect that MCS may play an important role in understanding the effects of other additives, such as surface passivating agents. Indeed, although few examples of this exist (section 2d), there is a large set of possible passivating inorganic and organic molecules that can easily be tested by MCS. Similarly, we have detailed a few results on non-perovskite multinary metal halides, including lead-free alternatives (section 3). These materials are highly 
sought after as lead toxicity remains one of the major drawbacks for a wider application of LHPs. Because MCS does not rely on solubility of precursors in organic solvents, which can be rather limiting for some metal halides, it appears as an ideal tool to study the plethora of lead-free multinary metal halides that have been theoretically proposed in the last few years as potential replacements for LHPs. In this sense, the few reports presented in section 3c, indicate that we have only scratched the surface on this topic. Eventually, the effective large-scale implementation of MCS in multinary metal halides' optoelectronics will be conditioned by the "processability" of the so-formed materials. To be more precise, the use of as-synthesized materials in devices such as solar cells or light-emitting diodes, relies on the ability to make high-quality thin films out of them. Most examples thereof so far have been conducted by redissolving the as-obtained powders in organic solvents. While functional devices have been obtained in this manner, using organic solvents for processing somehow defeats the purpose of using solvent-free MCS in the first place. Hence, single source vacuum deposition (SSVD) techniques have recently been proposed and demonstrated to yield phase-pure thin films of identical composition as the powder material obtained by MCS. This paves the way for a wider adoption of fully-dry synthetic methods in the development of LHPs and other multinary metal halides for optoelectronics.

\section{Acknowledgements}

The research leading to these results has received funding from the European Union Programme for Research and Innovation Horizon 2020 (2014-2020) under the Marie Skłodowska-Curie Grant Agreement PerovSAMs No. 747599, the Spanish Ministry of Economy and Competitiveness (MINECO) via the Unidad de Excelencia María de Maeztu MDM-2015-0538, MAT2017-88821-R and PCIN-2015-255, and the Generalitat Valenciana (Prometeo/2016/135 and GRISOLIAP/2017/089). H. J. B. acknowledges the support of ERA NET PCIN-2017-014. 
Received: ((will be filled in by the editorial staff))

Revised: ((will be filled in by the editorial staff))

Published online: ((will be filled in by the editorial staff))

\section{References:}

[1] D. Tan, F. García, Chem. Soc. Rev. 2019, 48, 2274.

[2] J. L. Do, T. Friščić, ACS Cent. Sci. 2017, 3, 13.

[3] M. K. Beyer, H. Clausen-Schaumann, Chem. Rev. 2005, 105, 2921.

[4] Q. Zhang, F. Saito, Adv. Powder Technol. 2012, 23, 523.

[5] J. L. Howard, Q. Cao, D. L. Browne, Chem. Sci. 2018, 9, 3080.

[6] M. Leonardi, M. Villacampa, J. C. Menéndez, Chem. Sci. 2018, 9, 2042.

[7] B. Gangdev, F. Patel, in Int. Conf. Multidiscip. Res. Pract., n.d., pp. 606-608.

[8] M. Klimakow, P. Klobes, A. F. Thünemann, K. Rademann, F. Emmerling, Chem. Mater. 2010, 22, 5216.

[9] D. Chen, J. Zhao, P. Zhang, S. Dai, Polyhedron 2019, 162, 59.

[10] P. Baláž, M. Achimovicová, M. Baláž, P. Billik, C. Z. Zara, J. M. Criado, F. Delogu, E. Dutková, E. Gaffet, F. J. Gotor, R. Kumar, I. Mitov, T. Rojac, M. Senna, A. Streletskii, W. C. Krystyna, Chem. Soc. Rev. 2013, 42, 7571.

[11] A. Urbieta, P. Fernández, J. Piqueras, J. Appl. Phys. 2004, 96, 2210.

[12] G. L. Tan, U. Hömmerich, D. Temple, N. Q. Wu, J. G. Zheng, G. Loutts, Scr. Mater. 2003, 48, 1469.

[13] L. Protesescu, S. Yakunin, O. Nazarenko, D. N. Dirin, M. V. Kovalenko, ACS Appl. Nano Mater. 2018, 1, 1300.

[14] J. Xing, Y. Zhao, M. Askerka, L. N. Quan, X. Gong, W. Zhao, J. Zhao, H. Tan, G. Long, L. Gao, Z. Yang, O. Voznyy, J. Tang, Z. H. Lu, Q. Xiong, E. H. Sargent, Nat. Commun. 2018, 9, 1.

[15] T. Leijtens, K. A. Bush, R. Prasanna, M. D. McGehee, Nat. Energy 2018, 3, 828. 
[16] R. Swartwout, M. T. Hoerantner, V. Bulović, Energy Environ. Mater. 2019, 2, 119.

[17] J. Ávila, C. Momblona, P. P. Boix, M. Sessolo, H. J. Bolink, Joule 2017, 1, 431.

[18] F. Palazon, D. Pérez-del-Rey, B. Dänekamp, C. Dreessen, M. Sessolo, P. P. Boix, H. J. Bolink, Adv. Mater. 2019, 1902692, 1.

[19] C. C. Stoumpos, C. D. Malliakas, M. G. Kanatzidis, Inorg. Chem. 2013, 52, 9019.

[20] D. Prochowicz, M. Franckevičius, A. M. Cië̈lak, S. M. Zakeeruddin, M. Grätzel, J. Lewiński, J. Mater. Chem. A 2015, 3, 20772.

[21] N. Leupold, K. Schötz, S. Cacovich, I. Bauer, M. Schultz, M. Daubinger, L. Kaiser, A. Rebai, J. Rousset, A. Köhler, P. Schulz, R. Moos, F. Panzer, ACS Appl. Mater. Interfaces 2019, 11, 30259.

[22] B. Dou, L. M. Wheeler, J. A. Christians, D. T. Moore, S. P. Harvey, J. J. Berry, F. S. Barnes, S. E. Shaheen, M. F. A. M. Van Hest, ACS Energy Lett. 2018, 3, 979.

[23] A. Jana, M. Mittal, A. Singla, S. Sapra, Chem. Commun. 2017, 53, 3046.

[24] D. J. Kubicki, D. Prochowicz, A. Hofstetter, M. Saski, P. Yadav, D. Bi, N. Pellet, J. Lewiński, S. M. Zakeeruddin, M. Grätzel, L. Emsley, J. Am. Chem. Soc. 2018, 140, 3345.

[25] V. A. Hintermayr, A. F. Richter, F. Ehrat, M. Döblinger, W. Vanderlinden, J. A. Sichert, Y. Tong, L. Polavarapu, J. Feldmann, A. S. Urban, Adv. Mater. 2016, 28, 9478.

[26] P. Pal, S. Saha, A. Banik, A. Sarkar, K. Biswas, Chem. - A Eur. J. 2018, 24, 1811.

[27] A. M. Askar, A. Karmakar, G. M. Bernard, M. Ha, V. V. Terskikh, B. D. Wiltshire, S. Patel, J. Fleet, K. Shankar, V. K. Michaelis, J. Phys. Chem. Lett. 2018, 9, 2671.

[28] A. Karmakar, A. M. Askar, G. M. Bernard, V. V. Terskikh, M. Ha, S. Patel, K. Shankar, V. K. Michaelis, Chem. Mater. 2018, 30, 2309.

[29] D. Chen, J. Li, X. Chen, J. Chen, J. Zhong, ACS Appl. Mater. Interfaces 2019, 11, 10059. 
[30] A. M. Elseman, A. E. Shalan, M. M. Rashad, A. M. Hassan, Mater. Sci. Semicond. Process. 2017, 66, 176.

[31] Y. El Ajjouri, V. S. Chirvony, M. Sessolo, F. Palazon, H. J. Bolink, RSC Adv. 2018, 8, 41548.

[32] O. Y. Posudievsky, N. V. Konoshchuk, A. G. Shkavro, V. L. Karbivskiy, V. G. Koshechko, V. D. Pokhodenko, ACS Appl. Nano Mater. 2018, 1, 4145.

[33] J. Breternitz, S. Levcenko, H. Hempel, G. Gurieva, A. Franz, A. Hoser, S. Schorr, J. Phys. Energy 2019, 1, 025003.

[34] D. J. Kubicki, D. Prochowicz, A. Hofstetter, S. M. Zakeeruddin, M. Grätzel, L. Emsley, J. Am. Chem. Soc. 2018, 140, 7232.

[35] D. Prochowicz, P. Yadav, M. Saliba, D. J. Kubicki, M. M. Tavakoli, S. M. Zakeeruddin, J. Lewiński, L. Emsley, M. Grätzel, Nano Energy 2018, 49, 523.

[36] M. M. Tavakoli, W. Tress, J. V. Milić, D. Kubicki, L. Emsley, M. Grätzel, Energy Environ. Sci. 2018, 11, 3310.

[37] M. Wilke, N. Casati, Chem. - A Eur. J. 2018, 24, 17701.

[38] S. Y. Kim, Y. Yun, S. Shin, J. H. Lee, Y. W. Heo, S. Lee, Scr. Mater. 2019, 166, 107.

[39] J. V. Milić, J. H. Im, D. J. Kubicki, A. Ummadisingu, J. Y. Seo, Y. Li, M. A. RuizPreciado, M. I. Dar, S. M. Zakeeruddin, L. Emsley, M. Grätzel, Adv. Energy Mater. 2019, 9, 1.

[40] N. Riesen, M. Lockrey, K. Badek, H. Riesen, Nanoscale 2019, 11, 4001.

[41] M. Saski, D. Prochowicz, W. Marynowski, J. Lewiński, Eur. J. Inorg. Chem. 2019, 2019, 2680.

[42] D. J. Kubicki, D. Prochowicz, A. Pinon, G. Stevanato, A. Hofstetter, S. M. Zakeeruddin, M. Grätzel, L. Emsley, J. Mater. Chem. A 2019, 7, 2326.

[43] D. Prochowicz, R. Runjhun, M. M. Tavakoli, P. Yadav, M. Saski, A. Q. Alanazi, D. J. Kubicki, Z. Kaszkur, S. M. Zakeeruddin, J. Lewiński, M. Grätzel, Chem. Mater. 2019, 
$31,1620$.

[44] A. D. Jodlowski, A. Yépez, R. Luque, L. Camacho, G. de Miguel, Angew. Chemie Int. Ed. 2016, 55, 14972.

[45] D. Prochowicz, P. Yadav, M. Saliba, M. Saski, S. M. Zakeeruddin, J. Lewiński, M. Grätzel, Sustain. Energy Fuels 2017, 1, 689.

[46] K. V. Manukyan, A. V. Yeghishyan, D. O. Moskovskikh, J. Kapaldo, A. Mintairov, A. S. Mukasyan, J. Mater. Sci. 2016, 51, 9123.

[47] Z. Y. Zhu, Q. Q. Yang, L. F. Gao, L. Zhang, A. Y. Shi, C. L. Sun, Q. Wang, H. L. Zhang, J. Phys. Chem. Lett. 2017, 8, 1610.

[48] P. Sadhukhan, S. Kundu, A. Roy, A. Ray, P. Maji, H. Dutta, S. K. Pradhan, S. Das, Cryst. Growth Des. 2018, 18, 3428.

[49] D. J. Kubicki, D. Prochowicz, A. Hofstetter, P. Péchy, S. M. Zakeeruddin, M. Grätzel, L. Emsley, J. Am. Chem. Soc. 2017, 139, 10055.

[50] O. Y. Posudievsky, N. V. Konoshchuk, V. L. Karbivskyy, O. P. Boiko, V. G. Koshechko, V. D. Pokhodenko, Theor. Exp. Chem. 2017, 53, 235.

[51] Y. El Ajjouri, F. Palazon, M. Sessolo, H. J. Bolink, Chem. Mater. 2018, 30, 7423.

[52] Y. El Ajjouri, V. S. Chirvony, N. Vassilyeva, M. Sessolo, F. Palazon, H. J. Bolink, J. Mater. Chem. C 2019, 7, 6236.

[53] G. Kaupp, CrystEngComm 2003, 5, 117.

[54] S. L. James, C. J. Adams, C. Bolm, D. Braga, P. Collier, T. Frišcic, F. Grepioni, K. D. M. Harris, G. Hyett, W. Jones, A. Krebs, J. Mack, L. Maini, A. G. Orpen, I. P. Parkin, W. C. Shearouse, J. W. Steed, D. C. Waddell, Chem. Soc. Rev. 2012, 41, 413.

[55] A. A. L. Michalchuk, I. A. Tumanov, S. Konar, S. A. J. Kimber, C. R. Pulham, E. V. Boldyreva, Adv. Sci. 2017, 4, DOI 10.1002/advs.201700132.

[56] A. A. L. Michalchuk, K. S. Hope, S. R. Kennedy, M. V. Blanco, E. V. Boldyreva, C. R. Pulham, Chem. Commun. 2018, 54, 4033. 
[57] A. A. L. Michalchuk, I. A. Tumanov, V. A. Drebushchak, E. V. Boldyreva, Faraday Discuss. 2014, 170, 311.

[58] Z. Hong, D. Tan, R. A. John, Y. K. E. Tay, Y. K. T. Ho, X. Zhao, T. C. Sum, N. Mathews, F. García, H. Sen Soo, iScience 2019, 16, 312.

[59] X. Lim, New York Times 2016, 1.

[60] R. A. Roufail, 2011, 266.

[61] A. Shrivastava, S. Sakthivel, B. Pitchumani, A. S. Rathore, Powder Technol. 2011, 211, 46.

[62] G. J. Lee, E. K. Park, S. A. Yang, J. J. Park, S. D. Bu, M. K. Lee, Sci. Rep. 2017, 7, 1.

[63] C. Frances, C. Laguérie, B. Mazzarotta, T. Veccia, Chem. Eng. J. Biochem. Eng. J. 1996, 63, 141.

[64] T. Tanaka, Adv. Powder Technol. 1995, 6, 125.

[65] A. Jankovic, Miner. Eng. 2003, 16, 337.

[66] F. Palazon, Y. El Ajjouri, P. Sebastiá, S. Lauciello, L. Manna, H. J. Bolink, J. Mater. Chem. $C$ n.d.

[67] G. Cravotto, E. C. Gaudino, P. Cintas, Chem. Soc. Rev. 2013, 42, 7521.

[68] G. Almeida, L. Goldoni, Q. Akkerman, Z. Dang, A. H. Khan, S. Marras, I. Moreels, L. Manna, ACS Nano 2018, 12, 1704.

[69] F. Palazon, G. Almeida, Q. A. Akkerman, L. De Trizio, Z. Dang, M. Prato, L. Manna, Chem. Mater. 2017, 29, 4167.

[70] S. Yun, A. Kirakosyan, S. G. Yoon, J. Choi, ACS Sustain. Chem. Eng. 2018, 6, 3733.

[71] A. Jana, M. Mittal, A. Singla, S. Sapra, Chem. Commun. 2017, 53, 3046.

[72] F. Meinardi, Q. A. Akkerman, F. Bruni, S. Park, M. Mauri, Z. Dang, L. Manna, S. Brovelli, ACS Energy Lett. 2017, 2, 2368.

[73] M. Saliba, T. Matsui, K. Domanski, J. Y. Seo, A. Ummadisingu, S. M. Zakeeruddin, J. P. Correa-Baena, W. R. Tress, A. Abate, A. Hagfeldt, M. Grätzel, Science (80-. ). 2016, 
354, 206.

[74] R. D. Shannon, Acta Cryst. 1976, A32, 751.

[75] G. Kieslich, S. Sun, A. K. Cheetham, Chem. Sci. 2015, 6, 3430.

[76] Z. Li, M. Yang, J. S. Park, S. H. Wei, J. J. Berry, K. Zhu, Chem. Mater. 2016, 28, 284.

[77] D. J. Kubicki, D. Prochowicz, A. Hofstetter, S. M. Zakeeruddin, M. Grätzel, L. Emsley, J. Am. Chem. Soc. 2017, 139, 14173.

[78] A. Karmakar, M. S. Dodd, X. Zhang, M. S. Oakley, M. Klobukowski, V. K. Michaelis, Chem. Commun. 2019, 55, 5079.

[79] M. Abdi-Jalebi, Z. Andaji-Garmaroudi, S. Cacovich, C. Stavrakas, B. Philippe, J. M. Richter, M. Alsari, E. P. Booker, E. M. Hutter, A. J. Pearson, S. Lilliu, T. J. Savenije, H. Rensmo, G. Divitini, C. Ducati, R. H. Friend, S. D. Stranks, Nature 2018, 555, 497.

[80] Y. El Ajjouri, F. Locardi, M. C. Gélvez-Rueda, M. Prato, M. Sessolo, M. Ferretti, F. C. Grozema, F. Palazon, H. J. Bolink, Energy Technol. 2019, ente. 201900788.

[81] D. Chen, J. Li, X. Chen, J. Chen, J. Zhong, ACS Appl. Mater. Interfaces 2019, 11, 10059.

[82] W. Xiang, Z. Wang, D. J. Kubicki, W. Tress, J. Luo, D. Prochowicz, S. Akin, L. Emsley, J. Zhou, G. Dietler, M. Grätzel, A. Hagfeldt, Joule 2019, 3, 205.

[83] M. J. Crane, D. M. Kroupa, J. Y. Roh, R. T. Anderson, M. D. Smith, D. R. Gamelin, ACS Appl. Energy Mater. 2019, 2, 4560.

[84] D. W. Dequilettes, S. Koch, S. Burke, R. K. Paranji, A. J. Shropshire, M. E. Ziffer, D. S. Ginger, ACS Energy Lett. 2016, 1, 438.

[85] C. C. Stoumpos, D. H. Cao, D. J. Clark, J. Young, J. M. Rondinelli, J. I. Jang, J. T. Hupp, M. G. Kanatzidis, Chem. Mater. 2016, 28, 2852.

[86] J. Yan, W. Qiu, G. Wu, P. Heremans, H. Chen, J. Mater. Chem. A 2018, 6, 11063.

[87] I. C. Smith, E. T. Hoke, D. Solis-Ibarra, M. D. McGehee, H. I. Karunadasa, Angew. Chemie - Int. Ed. 2014, 53, 11232. 
[88] D. H. Cao, C. C. Stoumpos, O. K. Farha, J. T. Hupp, M. G. Kanatzidis, J. Am. Chem. Soc. 2015, 137, 7843.

[89] G. Grancini, C. Roldán-Carmona, I. Zimmermann, E. Mosconi, X. Lee, D. Martineau, S. Narbey, F. Oswald, F. De Angelis, M. Graetzel, M. K. Nazeeruddin, Nat. Commun. 2017, 8, 1 .

[90] X. Li, J. Hoffman, W. Ke, M. Chen, H. Tsai, W. Nie, A. D. Mohite, M. Kepenekian, C. Katan, J. Even, M. R. Wasielewski, C. C. Stoumpos, M. G. Kanatzidis, J. Am. Chem. Soc. 2018, 140, 12226.

[91] E. Greul, M. L. Petrus, A. Binek, P. Docampo, T. Bein, J. Mater. Chem. A 2017, 5, 19972.

[92] A. Sarkar, P. Acharyya, R. Sasmal, P. Pal, S. S. Agasti, K. Biswas, Inorg. Chem. 2018, 57, 15558.

[93] K. Maji, P. Acharyya, P. Satapathy, S. K. Prasad, K. Biswas, Chem. - An Asian J. 2019, 1.

[94] N. Singhal, R. Chakraborty, P. Ghosh, A. Nag, Chem. - An Asian J. 2018, 13, 2085.

[95] D. Prochowicz, P. Yadav, M. Saliba, M. Saski, S. M. Zakeeruddin, J. Lewiński, M. Grätzel, ACS Appl. Mater. Interfaces 2017, 9, 28418.

[96] V. Ban, Y. Sadikin, M. Lange, N. Tumanov, Y. Filinchuk, R. Černý, N. Casati, Anal. Chem. 2017, 89, 13176.

[97] T. Friščić, I. Halasz, P. J. Beldon, A. M. Belenguer, F. Adams, S. A. J. Kimber, V. Honkimäki, R. E. Dinnebier, Nat. Chem. 2013, 5, 66.

[98] A. Goyal, S. McKechnie, D. Pashov, W. Tumas, M. Van Schilfgaarde, V. Stevanović, Chem. Mater. 2018, 30, 3920.

[99] G. Nedelcu, L. Protesescu, S. Yakunin, M. I. Bodnarchuk, M. J. Grotevent, M. V. Kovalenko, Nano Lett. 2015, 15, 5635. 
Table 1. References of phase-pure $\mathrm{APbX}_{3}$ LHPs prepared by dry MCS. MA = methylammonium $=\mathrm{CH}_{3} \mathrm{NH}_{3}{ }^{+} ; \mathrm{FA}=$ formamidinium $=\mathrm{CH}\left(\mathrm{NH}_{2}\right)_{2}{ }^{+}$. MCS of other multinary metal halides are discussed in following sections.

\begin{tabular}{c|ccc} 
& $\mathbf{X}=\mathbf{I}$ & $\mathbf{X}=\mathbf{B r}$ & $\mathbf{X}=\mathbf{C l}$ \\
\hline $\mathbf{A}=\mathbf{M A}$ & $\mathrm{MAPbI}_{3}^{[19,20,24,25,29,35,44]}$ & $\mathrm{MAPbBr}_{3}^{[25,29,35]}$ & $\mathrm{MAPbCl}_{3}{ }^{[25,29]}$ \\
$\mathbf{A}=\mathbf{F A}$ & $\mathrm{FAPbI}_{3}{ }^{[19,27,45,49,58,81]}$ & $\mathrm{FAPbBr}_{3}^{[27,29,35]}$ & $\mathrm{FAPbCl}_{3}^{[27,29]}$ \\
$\mathbf{A}=\mathbf{C s}$ & $\mathrm{CsPbI}_{3}{ }^{[19,26,58,77,81]}$ & $\mathrm{CsPbBr}_{3}^{[26,29,35,42]}$ & $\mathrm{CsPbCl}_{3}{ }^{[26,29,42]}$ \\
& & & \\
& &
\end{tabular}

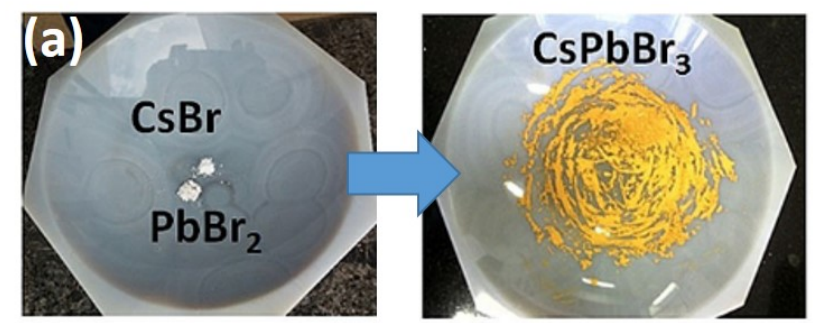

(b)
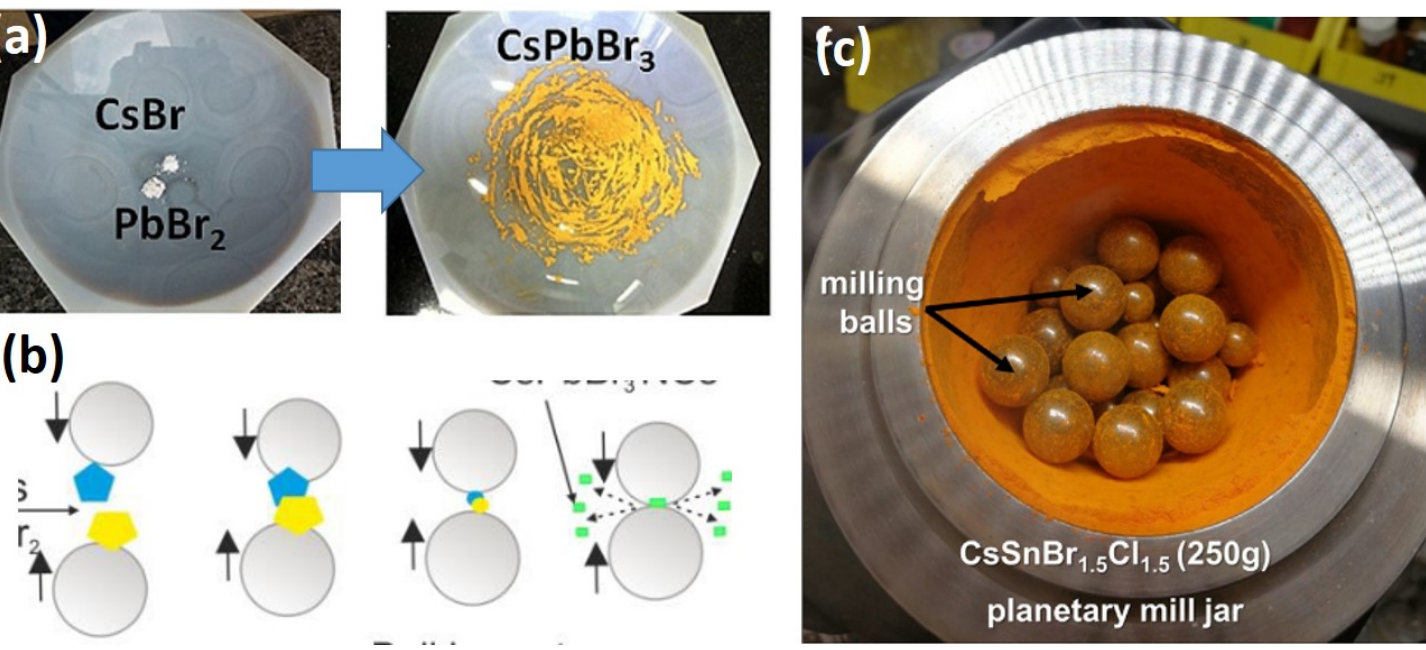

Figure 1. (a) photograph of mortar and pestle, adapted from Pal et al. ${ }^{[26]}$; (b) scheme of ballmilling, adapted from Protesescu et al. ${ }^{[13]}$; (c) Photograph of 250g mechanochemical synthesis, from Hong et al. ${ }^{[58]}$ 

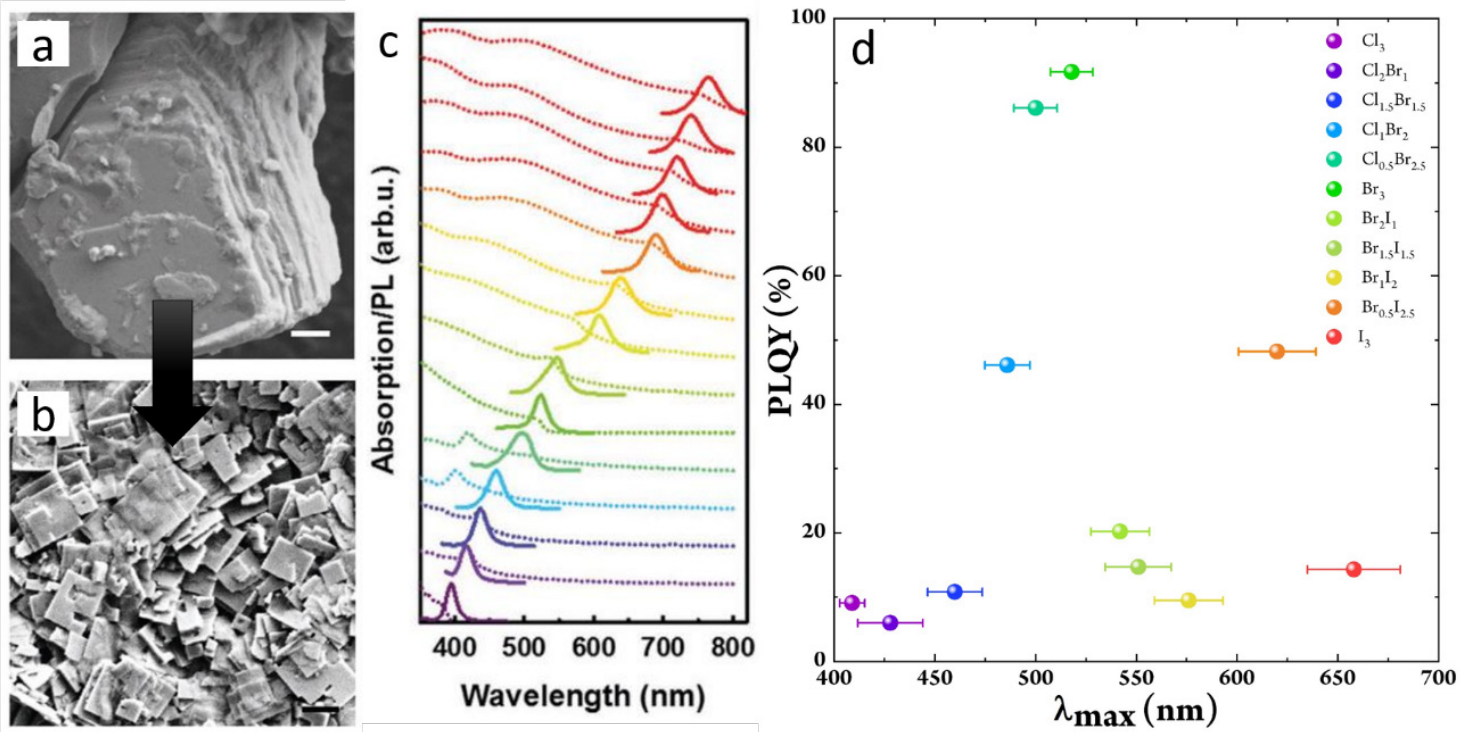

Figure 2. SEM images of "bulk" $\mathrm{MAPbX}_{3}$ crystal synthesized by dry grinding (a) and exfoliated nanoplatelets after tip sonication (b), adapted from Hintermayr et al. ${ }^{[25]}$ (c) Absorption (dotted lines) and PL (plain lines) of $\mathrm{MAPb}\left(\mathrm{Cl}_{x} \mathrm{Br}_{\mathrm{y}} \mathrm{I}_{\mathrm{z}}\right)$ synthesized by Hintermayr et al. ${ }^{[25]}$ (d) PLQY vs wavelength of CsPbX (X given in legend) obtained by Zhu et al. ${ }^{[47]}$ The lateral bars in (c) translate the FWHM of PL peaks. Scale bars in (a) and (b) are $100 \mathrm{~nm}$.
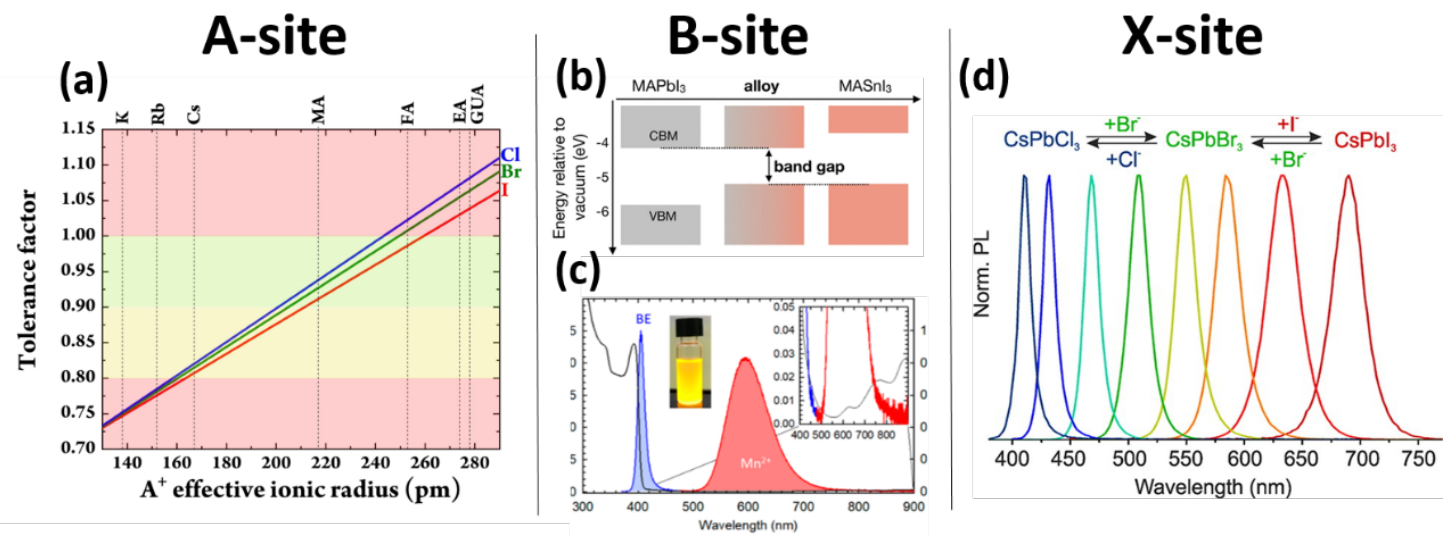

Figure 3. Overview of main reported effects of mixing different $\mathrm{A}, \mathrm{B}$, and $\mathrm{X}$ elements in $\mathrm{ABX}_{3}$ halide perovskites. (a) Tolerance factor of LHPs can be tuned by mixing A-cations (b) Mixed tin-lead iodide perovskites show lower bandgap than pure-tin and pure-lead counterparts; 
adapted from Goyal et al. ${ }^{[98]}$ (c) Mn-doped $\mathrm{CsPbCl}_{3}$ nanocrystals show bright PL with large Stokes-shift which can be used in luminescent solar cells concentrators; adapted from Meinardi et al., ${ }^{[72]}$ (d) Anion-exchange allows to tune the bandgap of halide perovskites across the whole visible range; adapted from Nedelcu et al. ${ }^{[99]}$ See main text for further explanations.

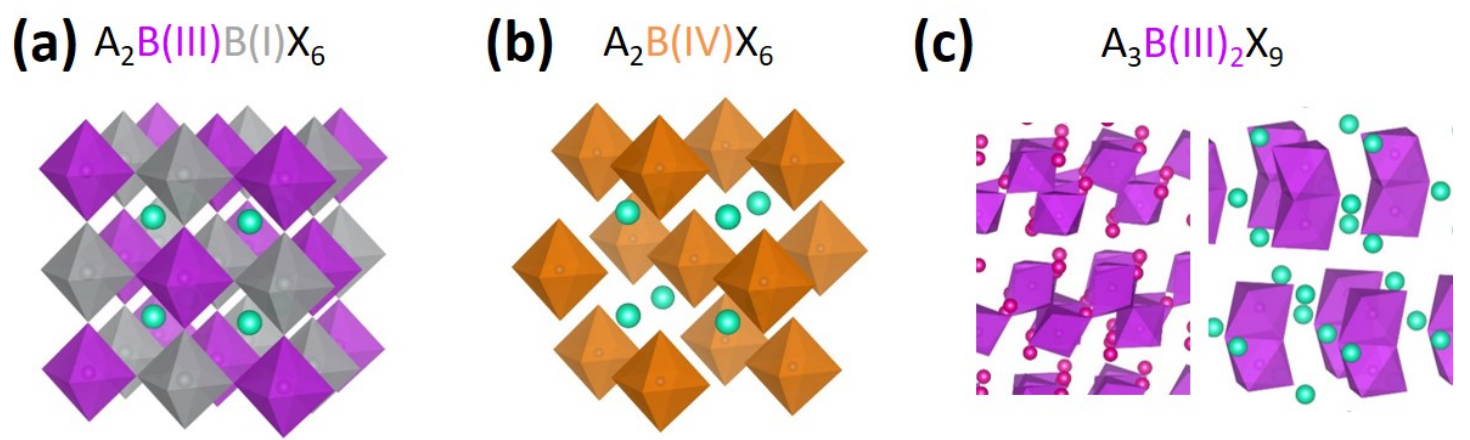

Figure 4. Crystal structures of different multinary metal halides. (a) elpasolite or "doubleperovskite” with a combination of monovalent and trivalent metal cations; (b) crystal structure obtained with a tetravalent cation such as Sn(IV); (c) different structures obtained with a trivalent cation such as $\mathrm{Bi}(\mathrm{III})$. Green balls represent “A” cations such as $\mathrm{Cs}^{+}$; color octahedra represent $\mathrm{BX}_{6}$ units. 


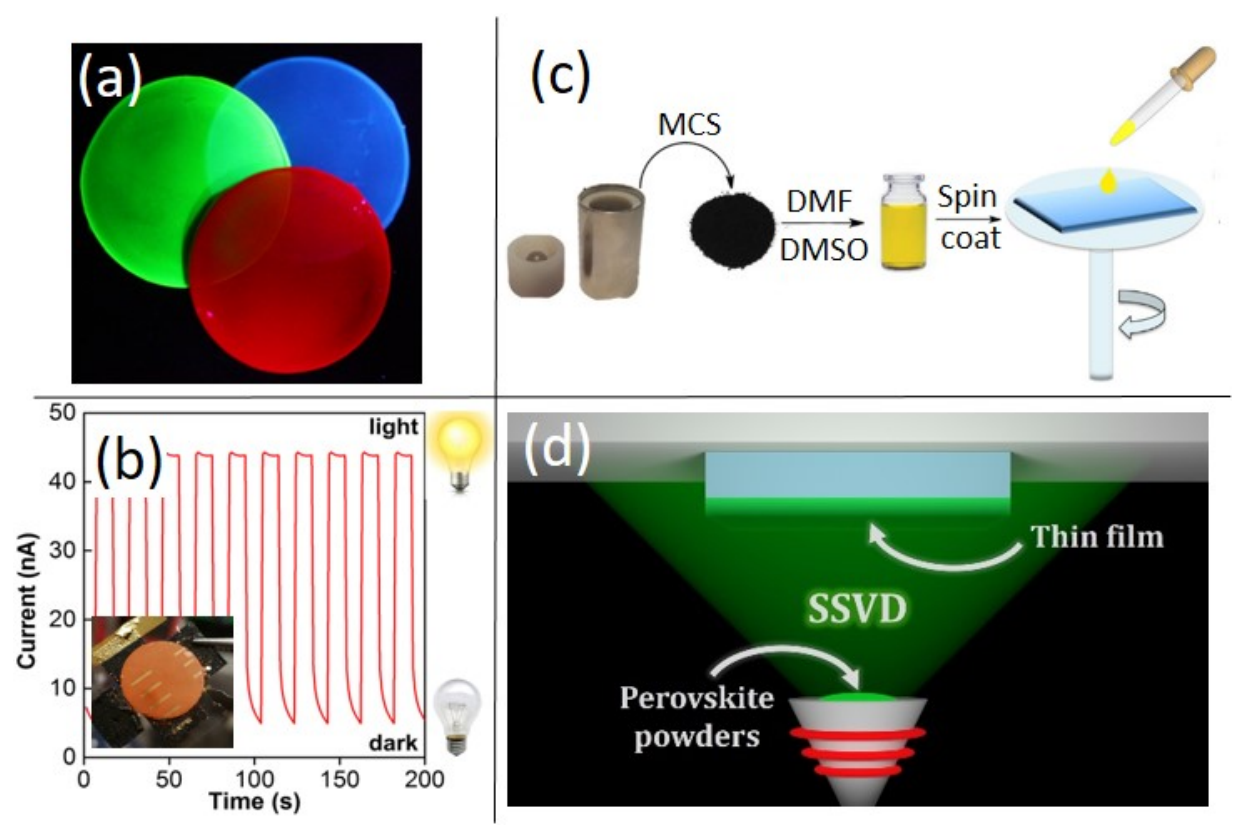

Figure 5. (a) QDs blended in PMMA for down conversion, adapted from Chen et al. ${ }^{[29]}$; (b) Photodetector made from a pellet of MCS-perovskites, adapted from Hong et al. ${ }^{[58]}$; (c) solution-processing of CsCl-containing perovskites prepared by MCS, adapted from Prochowicz et al. ${ }^{[35]}$; (d) fully-dry single-source vacuum deposition (SSVD) of perovskite thin films, adapted from El Ajjouri et al. ${ }^{[51]}$ 